\title{
Training Primary Care Physicians to Recognize and Manage Depression in Rural China: Dose It Relate to Their Subsequent Practices?
}

\author{
Jiang Xue ${ }^{1}$, Yeates Conwell ${ }^{2}$, Shulin Chen ${ }^{1}$ \\ ${ }^{1}$ Department of Psychology, Zhejiang University, China \\ 2Department of Psychiatry, University of Rochester, USA \\ Correspondence to Dr. Shulin Chen, MD, PhD, email: chenshulin@zju.edu.cn
}

Introduction: In response to the increasing burden of mental disorders, global initiatives call for the integration of mental health into primary care system (Collins, et al. 2013). However, it is frequently shown that primary care physicians (PCPs) do not always provide effective mental health services, especially in low-and-middle income countries. This study aimed to examine the efficacy of a training program on depression care management in PCPs in rural China, and to determine whether it relate to their subsequent depression care practices.

Methods: Data for the analysis were obtained from the Depression/HTN in Chinese Older Adults-Collaborations in Health $(\mathrm{COACH})$ Study, an ongoing project funded by the U. S. National Institute of Mental Health (NIMH) designed to compare collaborative depression care management with care as usual for the treatment of comorbid depression and hypertension in Chinese older rural village residents(Chen, et al. 2018).

During 2013 to 2015, 65 PCPs from rural villages in Tonglu County, China underwent a four-day training at the County Mental Hospital, and all of them completed a pre-post delivered survey on their knowledge of depression and self-efficacy toward depression management. In 2017, they were invited to undergo an online survey investigating their depression management practice in the past year. 60 PCPs (response rate of 92\%) completed the survey and were included in the analysis. All participants gave informed consent before participation.

Results: Demographic characteristics are listed in Table 1. The sample included 35 (58.3\%) females with a mean age of $43.7 \pm 14.2$ years old. All participants had received more than five years of education, and $28.3 \%$ of them had been working as PCPs for more than 10 years.

\begin{tabular}{|c|c|c|c|}
\hline Table 2 Learning outcomes of training & & \\
\hline Outcomes & Pre-score & Post-score & $p$-value \\
\hline Knowledge & & & \\
\hline $\begin{array}{c}\text { Symptoms of Dep } \\
\text { Myths of Dep }\end{array}$ & $3.08 \pm 0.42$ & $3.30 \pm 0.42$ & 0.005 \\
\hline Responding to Dep & $2.08 \pm 0.60$ & $2.84 \pm 0.77$ & $<0.001$ \\
\hline Self-efficacy & $2.24 \pm 0.42$ & $2.83 \pm 0.57$ & $<0.001$ \\
\hline Listening/Asking about Dep & $2.61 \pm 0.60$ & $2.83 \pm 0.53$ & 0.023 \\
\hline Recognizing Dep & $2.18 \pm 0.60$ & $2.83 \pm 0.62$ & $<0.001$ \\
\hline Communicating to seniors & $2.27 \pm 0.68$ & $2.74 \pm 0.66$ & $<0.001$ \\
\hline
\end{tabular}

Supported by US National Institutes of Mental Health, Grant Number:R01MH100298
After trained, PCPs achieved significant improvements on each aspects of their knowledge on depression and self-efficacy on working with depressed patients (Table 2).

Both knowledge $(p=0.046)$ and self-efficacy $(p=0.007)$ change were found to significantly predict one's depression screening, while only the latter $(p=0.005)$ significantly predict the frequency of consulting a psychiatrist (Table 3 ).

Conclusion: The training program is effective in improving PCPs' knowledge and self-efficacy on depression care management, which are significantly associated with their subsequent depression care practices. More and more appropriate and sufficient training should be delivered in order to improve PCPs' capability of providing effective mental health services.

\begin{tabular}{|c|c|c|}
\hline Demographic characteristics & $\mathrm{N}$ & $\%$ \\
\hline \multicolumn{3}{|l|}{ Age } \\
\hline $20-39$ & 29 & 48.3 \\
\hline $40-59$ & 18 & 30.0 \\
\hline 60 or more & 13 & 21.7 \\
\hline Female & 35 & 58.3 \\
\hline \multicolumn{3}{|l|}{ Education } \\
\hline $0-5$ years & 0 & 0.0 \\
\hline $6-8$ years & 7 & 11.7 \\
\hline $9-11$ years & 32 & 53.3 \\
\hline$\geq 12$ years & 21 & 35.0 \\
\hline \multicolumn{3}{|l|}{ Years as a PCP } \\
\hline $1-9$ & 43 & 71.7 \\
\hline$\geq 10$ & 17 & 28.3 \\
\hline Doing depression screening & 50 & 83.3 \\
\hline \multicolumn{3}{|l|}{ Consulting a psychiatrist } \\
\hline Once per month & 8 & 13.3 \\
\hline Once every 2 or 3 months & 28 & 46.7 \\
\hline 2 or 3 times per year & 24 & 40.0 \\
\hline \multicolumn{3}{|l|}{ Confident in prescribing antidepressants } \\
\hline Not confident & 42 & 70 \\
\hline Confident) & 18 & 30 \\
\hline
\end{tabular}

\begin{tabular}{|c|c|c|c|c|c|}
\hline Behavior & variable & B & S.E. & Wald & Sig. \\
\hline \multirow[t]{5}{*}{ Depression screening } & Age & -0.142 & 0.090 & 2.487 & 0.115 \\
\hline & Years as a PCP & 0.179 & 0.103 & 3.032 & 0.082 \\
\hline & Post knowledge & -0.679 & 0.387 & 3.075 & $0.08 \mathrm{c}$ \\
\hline & Knowledge change & 0.671 & 0.336 & 3.981 & $0.04 €$ \\
\hline & Confidence change & 0.577 & 0.214 & 7.283 & 0.007 \\
\hline \multicolumn{6}{|l|}{ Consulting case $\mathrm{e}^{\mathrm{a}}$} \\
\hline \multirow[t]{3}{*}{ Once per month } & Years as a PCP & -0.129 & 0.096 & 1.826 & 0.177 \\
\hline & Knowledge change & 0.300 & 0.156 & 3.693 & 0.05 \\
\hline & Confidence change & 0.256 & 0.091 & 7.959 & 0.005 \\
\hline \multirow[t]{3}{*}{ Once 2 or 3 months } & Years as a PCP & -0.214 & 0.079 & 7.317 & 0.007 \\
\hline & Knowledge change & 0.103 & 0.087 & 1.401 & 0.236 \\
\hline & Confidence change & 0.077 & 0.060 & 1.669 & 0.196 \\
\hline \multicolumn{6}{|l|}{ Confidence in prescribing ${ }^{\mathrm{b}}$} \\
\hline $2^{c}$ & Years as a PCP & -0.109 & 0.056 & 3.712 & 0.05 \\
\hline $3^{c}$ & Years as a PCP & -0.131 & 0.060 & 4.816 & $0.02 \varepsilon$ \\
\hline $4^{c}$ & Years as a PCP & -0.183 & 0.163 & 1.253 & 0.263 \\
\hline
\end{tabular}

Copyright @ 2017 Shulin Chen: chenshulin@zju.edu.cn 